\section{Europe's mental health support}

$\mathrm{H}$ ow does Europe fare as far as mental healthcare provision is concerned? Thirty-eight per cent of Europeans suffer from a mental illness every year. In 2014, the Intelligence Unit of the Economist, sponsored by Janssen Pharmaceutica $\mathrm{NV}$, carried out a study and produced a report titled Mental Health Integration - Provision for Supporting People With Mental Illness: A Comparison of 30 European Countries, which makes sobering reading. The study examined a number of measures, including environment (providing a stable home and family), access to healthcare, opportunities for improving work and education, and what was termed 'governance' (reducing stigma and increasing awareness). Despite some differences across the 30 European countries, the common themes were "silo thinking and acting and lack of integrated support'. The report identified a number of areas where action was needed: more research into the epidemiological, medical and social care process and outcomes; availability of funding 'appropriate to the task'; finishing the task of deinstitutionalisation; focusing on the task of providing integrated, community-based services; and integrated employment services in community-based care provision.

\section{No health without mental health}

Where is already a large body of evidence demonstrating that mental illness is associated with high rates of physical morbidity and mortality. A meta-analysis of 203 studies from 29 countries carried out by researchers from Emory University in Atlanta estimated the risk of death among those with mental illness (from 148 studies) to be 2.22 times higher than in the comparison population. Of the deaths among the mentally ill group, $67.3 \%$ were due to natural causes and $17.5 \%$ to unnatural causes, with the rest attributed to 'other or unknown causes'. It was estimated that $14.3 \%$ of deaths worldwide, that is about 8 million deaths per year, are attributable to mental disorders and it was concluded that more attention should be paid to the more common mental disorders, with emphasis on preventing and managing comorbidity with physical conditions.

Reisinger, E., et al (2015) Mortality in mental disorders and global disease burden implications. JAMA Psychiatry. doi: 10.1001/ jamapsychiatry.2014.2502.

\section{'Sestrin 3', the epilepsy gene, orchestra conductor?}

ost neuropsychiatric conditions are attrib1 uted to multiple gene involvement but how does this gene network operate? Researchers at Imperial College London used a technique called 'systems genetics' to unravel how genes work together in epilepsy. They examined brain tissue donated by 129 people and carried out further analyses using laboratory mice and zebra fish. They were able to identify a gene known as Sestrin 3 (SESN3), which had never been linked to epilepsy before but which is involved in coordinating about
400 genes associated with epilepsy. Unravelling how SESN3 controls the gene network could lead to the development of more effective anti-epileptic agents. Understanding the mechanisms involved in gene regulation in epilepsy could also be useful in the management of other brain conditions, such as Alzheimer's disease and neurodevelopmental disorders.

Johnson, M., et al (2015) Systems-genetics identifies Sestrin 3 as a regulator of a proconvulsant gene network in human epileptic hippocampus. Nature Communications, 26 January. doi: 10.1038/ ncomms7031.

\section{Chronic undernourishment}

s many as 805 million people, that is one in A many as 805 million people, that is one in
nine people in the world (total world population 7.3 billion), are considered chronically undernourished according to the United Nations Food and Agriculture Organization. Most of these (791 million) live in low- and middle-income countries, and poverty plays a major role in their undernourishment. However, living in affluent countries doesn't guarantee adequate nourishment; as many as 11 million people who live in high-income countries are also considered undernourished! You can read more on the subject of world hunger and malnutrition and their causes at http://www.fao.org/publications/sofi/2014/en/

\section{Good night!}

Tnsomnia, a common condition on its own or in association with other conditions (psychiatric or physical), is often a 'nightmare' to treat for doctors and a major burden to patients. Several generations of hypnotics have, over the years, been claimed to be better than their predecessors but they all eventually were found to cause tolerance and dependence, which impose major limitations in their use. Better understanding of how the brain works in inducing sleep could pave the way to the development of more specific and non-dependence-forming drugs. It was believed that hypnotics acting via particular receptors (e.g. GABA) have a wide effect on all parts of the brain. However, widespread action on the brain may not be necessary, according to scientists at Imperial College London, who made an interesting discovery. They showed that 'switching on' the neurons in a specific area of the brain, the preoptic hypothalamus, is responsible for shutting down the areas of the brain that are inactive during sleep. This process is very similar to what happens in the 'deep recovery sleep' which occurs after a period of sleep deprivation. They demonstrated that certain types of sedative drugs work by switching on this area of the brain. Could this mean that new drugs which target these neurons in the preoptic hypothalamus will be successful in inducing sleep in a more 'natural' way and be less likely to be associated with dependence problems?

Zhang. Z., et al (2015) Neuronal ensembles sufficient for recovery sleep and the sedative actions of $\alpha_{2}$ adrenergic agonists. Nature Neuroscience. doi: 10.1038/nn.3957. 